\title{
Suicidal Behaviour in Complex Trauma Syndrome
}

\author{
Vito Z* \\ Psychclinic P/L, London, UK
}

Research Article

Volume 4 Issue 1

Received Date: November 24, 2019

Published Date: January 04, 2020

DOI: $10.23880 / \mathrm{mhrij}-16000136$

\section{Abstract}

Suicide is a phenomenon which remains among the most tragic consequences of any mental disorder and the assessment of suicidal potential (suicidal ideation, suicide attempt, self-harm, etc.) should be of the highest clinical priority to treat. Identifying those individuals who are at a high risk for attempting suicide or self-harm expands the clinician's reference in assessing and treating suicidal patients more seriously than any other mental health problem. Suicidality is not a diagnostic criterion for PTSD, however, in severe and complex cases of the psychological trauma this problem appears quite frequently. In this paper, we have elaborated suicidal behaviour of 36 trauma syndrome patients (complex PTSD) using psychometric instruments (BHS, CAPS, SCID) and clinical interview. Our results demonstrate a high incidence of suicidal behaviour in severely traumatised individuals.

Keywords: Complex Trauma Syndrome; Suicide; Self-at-Worst; Dysfunctional Appraisals; Hopelessness

\section{Introduction}

Although suicidal behaviour is not diagnostic criterion for PTSD, there is general consensus among clinicians about a strong association between psychological trauma and suicidal behaviour. Several studies have examined factors which confirmed that those diagnosed with complex PTSD are under suicidal risk [1-3]. These studies have demonstrated that suicidal risk is significantly and positively associated with one's re-experiencing symptoms cluster, hyperarousal, and negatively or insignificantly associated with the avoidance and numbing symptom cluster. However, the existing literature examining associations between PTSD and suicidal behaviour did not provide enough evidence elucidation the mechanisms by which a suicidal behaviour develops from the PTSD. One possibility for this clinical puzzle is that particular symptoms of the PTSD leads to depression, which then leads to the patient's suicidal behaviour [2-4]. Further, one's comorbid depression may lead to suicidal behaviour because it exacerbates the effects of PTSD symptoms.

Regardless of causal factors, suicidal risk represents a serious public health problem and should be prioritise in prevention and treatment. Identifying characteristics of patient at a high risk for attempting or committing suicide expands the clinician's reference base in assessing suicidal potential. Familiarity with risk factors for suicidal behaviour helps the clinician to register the import of the data from the clinical interview and/or psychometric assessment and make a more cogent appraisal of the possibility for suicide. Suicidal risk refers a range of patient's thoughts, from an idea that the death would be welcome, to the immediate intent/attempt to commit suicide. Thus, suicide remains among the most dreaded outcomes of psychiatric disorder and commonly 


\section{Mental Health \& Human Resilience International Journal}

represents salient issues of the patient's problems. Treating patients with severe psychiatric disorders (e.g., personality disorders, major depression, complex trauma syndrome, etc.), the clinician should always assess any potentiality of suicidality [5]. This includes the assessment of suicidal potential in the patient who has already engaged in a suicide attempt, as well as a delineation of the contribution of psychological stressors (past and/or current), affective, personality and cognitive style, intercurrent medical illness, and specific psychiatric syndromes which may lead to a suicidal risk in psychiatric patients.

Suicidal risk should be assessed initially and over the course of therapy. If at any stage patient shows suicidal attempt, ideation, intention, or plan, close surveillance is necessary and mandatory. The risk of suicide in some patients recovering from trauma syndrome increases transiently as they develop the energy and capacity to act on self-destructive plans which had been present already in the course of disorder [3]. Clinicians who deal with trauma syndrome patients should be aware of the risk of suicide throughout the course of treatment. However, the prediction of suicide attempts or suicide completion for any given patient is extremely difficult because of (1) false positives during the treatment (patients who appear to be at greater suicidal risk but who do not) and (2) false negatives (patients who appeared to be at decreased suicidal risk but who ultimately do attempt or committed suicide, or do self-harm).

An assessment of the presence and extent of suicidality in severely traumatised individuals is an essential part of the psychiatric (psychological) examination. Suicidality in complex trauma syndrome exists as a spectrum from nonspecific suicidal ideation, such as thoughts of death, to thoughts of one's own death, and finally to suicidal thoughts with a plan and intent to commit suicide. It is common that the severely traumatised individuals suffer more severe forms of suicidal ideation and often represents a silent danger which needs an urgent approach. The psychological trauma consequently impacts all aspects of the selfstructure-one's image of the body; the internalised images about others; and one's values and ideals - and leads to a sense that self-coherence and self's goals are invaded, assaulted and systematically broken down [6]. Chronic trauma causes identity diffusion, fragility and feelings of self-discontinuity, with severely disrupted/shattered selfcohesion, interpersonal relationships, and existence. Severely traumatised individuals may find suicide as an 'emergency solution' in order to escape from the 'persistence of trauma' that continues a long into the posttrauma time.

Traumatic events overwhelm an ordinary human adaptation to life and generally involve threat to life, or bodily integrity. A close personal encounter with severe violence, threat and death, confronts trauma victims with the extremities of hopelessness, disconnection, terror, disempowerment, and evoke the response of inescapable catastrophe. Restoring feelings of the self-cohesiveness is not an easy task for patients, especially with the victims of the human-design aggression and violence (e.g. combat, terrorism, severe rape). The vulnerable self-structure of the severely traumatised individuals could be evidenced in the following aspects: (1) difficulties in self-regulation, such as self-esteem maintenance, affect tolerance, sense of the self-cohesion and continuity, or the sense of personal agency; (2) appearance of stress symptoms, such as frequent urges of fears and anxiety, depression, or irritability; and (3) the individual's reliance on primitive or less-developed forms of the self-object relatedness with attachment figures [5].

When one's basis of the self-structure and its organisation are damaged by the trauma, the aftermaths of the trauma may occur in various forms and degrees of the self-dissolution, fragmentation, disintegration, and self-destructiveness. This prolonged trauma-related condition we emphasised as a distal exposure to the event(s) and consequences which manifest most clearly in the aftermaths of a disaster. Severely traumatised individual who is suicidal presents his 'self-at-worst' state compromised without a safety net or an access to the emotional resources. In terms of practical reality and given the complexities in the ways that trauma unfolds over time, we assume that the perceived-life-threat aspects of the trauma are often of the strongest predictors in the risk of suicide for a trauma survivor [4].

There are numerous studies which revealed that survivors of prolonged, repeated trauma (such as childhood trauma, brutal rape) suffer complex trauma syndrome and became psychiatric patients. Their general levels of severe distress are higher than those with a simple stress disorder (e.g. car accident, natural disaster). They usually have significantly higher scores on the standardised measures of somatisation, dissociation, depression, phobic anxiety (fears), interpersonal sensitivity, and depleted sense of the self and selfcontinuity [4]. They also display significantly more anger, insomnia, sexual dysfunctions, suicidal behaviour, selfmutilation, repeated victimisation, drug addiction, and alcoholism. Prolonged or repeated traumas usually cause 


\section{Mental Health \& Human Resilience International Journal}

symptoms list almost indefinitely and life-lasting. Survivors of childhood abuse or brutal rape, like other traumatised individuals, are frequently misdiagnosed and mistreated in particular with their evident suicidal behaviour - because of the number and complexity of the symptoms; their treatment is often fragmented and incomplete. They often develop fragmented identity, altered state of consciousness, detachment, memory lapses, and other dissociative symptoms.

Depleted or shattered self-had been afflicted with a sense of emptiness in painful intensity of the traumatic memories, leading to a suicide as desperate attempt to 'fill a gap', which imprints even further destructions of the self as it has 'nothing inside' $[7,8]$. Severely traumatised individuals describe their loss of the self-structure in different ways - they feel they are falling apart, losing their bearings, or 'treading water in the middle of the ocean with nothing to hang on to', they may feel lost in space, or even feel non-existing [6]. For the clinicians, it is significant to recognise patient's use of negative terms while describing an experience of the fragmentation of the mind-body-self $[9,10]$. In some extreme cases of dissociation caused by trauma, the patients would report dissociated parts of the body and that their body has become 'stranger' of 'foreign' to them [4].

Trauma may lead to a sense of de-centering of the self, loss of groundedness and a sense of sameness, continuity of the ego-fragility, leaving scars on the one's inneragency of the psyche [6]. In many cases it is apparent that fragmentation of the self-identity is a fracture of the soul and spirit of the person, like a broken connection of the patient's sense of the existence. Any fragmentation of the self-identity has huge consequences in the patient's psychological stability, his well-being, and psychic integration resulting in a proneness to disintegrated personality as a whole [6,11-13].

The main aim of this study was to formulate risks to the suicidal behaviour in patients with complex trauma syndrome. The formulation of 'complex trauma' or 'complex PTSD' has been present in the literature and the clinical practice for few decades but still is not been diagnostically determined either in the DSM or ICD. It is out of scope of this paper to review the inconsistency between the clinical practice and the diagnostic references, but indeed, some points should be mentioned in this paper as well.

Clinicians are agreed that the complex PTSD is the trauma spectrum (syndrome) or a distinct entity, rather than a single diagnostic entity [14-18]. Herman [18] stated that studies of subjects with histories of trauma revealed the lifetime prevalence of the complex PTSD much higher than the PTSD alone. Further, it is documented [6,17] that complex PTSD is more accountable, than the PTSD alone, for the patient's functional impairments and multiple comorbidities (e.g., affect dysregulation, disintegration of the self-structure, and problems in interpersonal relationships). In this paper we indicated complex trauma to those PTSD patients who were exposed to the severe repeated and/or prolonged traumatic event(s) causing severity of clinical symptoms and/or the negative perceptions of functional impairments which makes a risk for patient's suicidal behaviour.

\section{Methods}

\section{Participants}

Assessing suicide risk is a complex task which nearly every professional in mental health area faces in his/her clinical and research practice. Clinically, the suicide should not be considered as a single entity and thorough assessment of this puzzling problem should be idiographic and attempt to assess the various points along the continuum from one's ideation to attempt or to complete suicide. The goals of any clinical assessment include screening, description of the symptoms associated with suicide and primary diagnosis of mental disorder(s), determination of the symptoms' severity, evaluation of contributing factors to the patient's distress as a means of assisting with case formulation, monitoring of treatment responses and gains, and prediction of suicidal behaviour.

Prediction of suicide risk is among the most important and challenging tasks confronting clinician who faces with multifactorial factors (ideation, gestures, attempt, and (perhaps) completed suicide). Suicidality is not diagnostic entity either in the DSM or ICD but requires multimodal approach to review diagnosis of mental disorder(s) (e.g., depression, PTSD, schizophrenia), hopelessness or pessimism, the social-problem solving deficits, cognitive distortions, and dysfunctional schema or attitudes; all of these could indicated one suicidal behaviour.

Data from 36 patients (21 male and 15 female) attended a screening process due to their severe traumatic experience(s) which led to a complex trauma syndrome (which includes potential risk for suicide). The participants were referred for treatment from primary and secondary health services because of the severity and complexity of their condition, often showing clinical symptoms which likely being treatment resistant. Those 


\section{Mental Health \& Human Resilience International Journal}

patients initially screened by the Beck Hopelessness Scale (BHS) [19] who scored between 15-20 points (severe hopelessness) were then further interviewed by the clinician regarding their suicidality. The aims of using the $B H S$, as an aid to suicide assessment, were: (1) to identify specific factors and features that generally indicates risk for the suicide or other patient's suicidal behaviours; (2) address the patient's immediate safety and determine the most appropriate setting for treatment; and (3) to develop a multiaxial differential diagnosis to further guide planning or treatment.

The inclusion criteria in this study were a diagnosis of PTSD according to the Diagnostic and Statistical Manual of Mental Disorder (DSM-5) [20], confirmed by the results on Clinician-Administered PTSD Scale (CAPS) [21], and the clinician's report after an interview with the patients. Further inclusion criteria were a minimum of six months being diagnosed with PTSD and not suffering from a psychotic or organic brain illness, nor primary diagnosis of alcohol or substance abuse; and not younger than 18 or older than 60 years of age.

\section{Measures}

The $\boldsymbol{B} \boldsymbol{H} \boldsymbol{S}$ is self-administered test designed to measure hopelessness as cognitive schemata of the negative expectancy about the short-term and log-term future. It is designed to measure the extent of negative attitudes and thoughts about the future (pessimism) as it is perceived by patient. The BHS originally was not designed as a screening instrument, but it has nevertheless been used as an indirect indicator of the suicide risk in the depressed individuals who have made suicide attempts, and for detection of hopelessness. The final version of the $B H S$ consists of 20 true-false statements. In its original validation study, a sample of 294 hospitalised patients who made a recent suicide attempt served as the sample. When data from the 294 suicide attempters were subjected to the factor analysis, three factors were emerged: feelings about the future, loss of motivation, and future expectations. The reliability coefficient was 0.93 , and an item-total correlation coefficient ranged from 0.39-0.76.

The scale is used in inpatient, outpatient, and emergency psychiatric settings. It contains 20 items and the severity of hopelessness is calculated by summing the scores [19]. It is recommended classification of the scores as follow: 0-3 minimal, 4-8 mild, 9-14 moderate, and 1520 severe hopelessness. The $B H S$ is positively associated with elevated risk of any eventual suicide attempt or suicide completion. High scores (15-20) we found indicate that the further assessment and treatment of the patients should be taken.

Beck [19] did not suggest that any high score is ultimately high risk for suicide - high score for individual does not have clinical utility in predicting immediate risk of suicide attempt. It is a screening test and high score indicates needs to monitor patient due to potentials for suicide and needs to take early interventions to reduce some eventual suicidal attempt or completion. Therefore, the suicide assessment scales may be used as aids to suicide assessment but should not be used as predictable instruments, or as substitution for a thorough clinical evaluation.

In our research, after evaluation of the BHS, the clinician further evaluated a risk of suicide in the clinical interview questioning patient regarding suicidal thoughts (e.g., 'Have you seriously thought about suicide?'), suicidal attempts (e.g., 'Have you ever attempted suicide or attempted to end your life?'), and/or suicidal plans (e.g., 'Have you ever made plans to commit suicide?'). The clinical interview was targeting consistency between scores on the BHS and one's real risk, thoughts, or attempt for suicide. Hopelessness has received strong empirical support as a risk factor for suicide and prospective studies of both inpatients and outpatients and BHS became a predictive utility.

Although every patient has been diagnosed of suffering PTSD more than six months (most of them already being prescribed with medication) the use of CAPS was aimed to measure severity and complexity of the PTSD symptoms. In fact, the CAPS is a structured interview scale developed to assess the type of severity of posttraumatic stress disorder symptoms. Although the original scale was designed to assess a combat-related PTSD it is useful to assess severity and frequency of symptoms of both combat and civilian trauma. Initially, it was assumed that the CAPS total score is associated with both severity of the trauma syndrome symptoms and social functioning impairment.

The CAPS consist of 17 interviewer-related items which cover main core of posttraumatic disorder defined in the DSM criteria, including re-experiencing of the traumatic event, corresponding to the posttraumatic stress disorder Criterion $B$ (e.g., intrusive distressing memories, distressing dreams, dissociative reactions (flashbacks), intense or prolonged psychological distress at exposure to internal or external cues that symbolise an aspect of the traumatic event). Also, it corresponds with the Criterion $C$ (e.g., persistent avoidance of stimuli 


\section{Mental Health \& Human Resilience International Journal}

associated with the traumatic event(s), the avoidance of distressing memories, thoughts, places, feelings, people, activities, situations, etc.), and the Criterion $E$ which defines one's marked alterations in arousal and reactivity associated with the traumatic event(s), (e.g., selfdestructive behaviour, hypervigilance, exaggerated startle response, problems with concentration, irritable behaviour, sleep problems).

Eight additional items are included to measure the frequency and intensity of features associated to the Criterion $D$ (negative alterations in cognitions and mood associated with the traumatic event(s)). While using the $C A P S$, the clinician is free to evaluate patient's cognitive functioning, persistence of the negative beliefs or expectations about oneself, persistent negative emotional state (e.g., fear, horror, anger, guilt, or shame), feelings of detachment or estrangement from others, markedly diminished interest in significant activities, inability to experience positive emotions. In addition, the CAPS includes five global rating scales that reflects the impact symptoms on a social and occupational functioning, general severity, any recent changes in severity, and the clinician's evaluation of the validity of the patient's report.

For each of the 17 items, the interviewer rates the frequency of symptoms $(0=$ never, $1=$ once or twice, $2=$ once or twice a week, $3=$ several times a week, and $4=$ daily or almost every day $)$ and the intensity $(0=$ none, $1=$ mild, $2=$ moderate, $3=$ severe, and $4=$ extreme) of the symptoms (e.g., Can you describe your thoughts at the time that you were thinking most seriously about suicide? At its worst, how much did it seem that the event was happening again? How long did it last? What did you do while this was happening?). Thus, the score ranges from 0 to 8 per item and maximal score could be 136 for all 17 items. The CAPS interview can be scored as per DSM criteria for posttraumatic stress disorder, rendering a dichotomous rating a presence or an absence of the stress disorder.

The manual should be used by trained clinician who is familiar not only with PTSD but also with other associated stress-related disorders [21]. It is not necessary to have a high skill of statistical analysis as evaluation of the CAPS scale results is quite simple and routine. The CAPS interview has high internal consistency; Cronbach's alphas for the individual subscale and total score ranged $0.70-0.88$. Because of its practicality, the CAPS is a worldwide used instrument and has been translated in world's most leading languages. The instrument is also useful and appears sensitive to symptoms improvement with psychological treatment and spontaneous mission. An assessment by the CAPS interview takes approximately forty-five to sixty minutes, although administration time can be decreased if administered on the same patient repeatedly.

To assess more accurately suicidality among the severely traumatised individuals we also used Structured Clinical Interview (SCID), which is probably the most reliable approach to evaluate one's specific aspects of suicidal thoughts, plans, and behaviour related to the severity and the complexity of PTSD. The PTSD version of the $S C I D$ can be administered independently in the context of full assessment of PTSD or with other versions to assess other stress-related disorders [22]. Administration of additional modules assesses the disorders which are highly comorbid with the PTSD, such as depression or personality disorders, giving more comprehensive description and validation of one's problems. The SCID modules directly cover all PTSD symptoms included in the DSM-5 diagnostic criteria, including assessment of dissociation. The modular structure of the SCID allows clinician to limit assessment to condition that is comorbid with the PTSD, however, more accurate assessment of the severity and complexity of trauma could be in using open question interview which delivers other details about one's traumatic experience apart from the SCID sections.

The clinical interview can be divided into inquiries about patient's feelings about living which could be covered by questions like 'Have you ever felt that life is not worth living?' or 'Did you ever wish you could go to sleep and just not wake up?'. The severely traumatised individuals are usually reluctant to talk about their past and feelings, however, the clinician should reach good rapport and encourage patient to openly talk about feelings, thoughts, relationships, and behaviour.

When suicidal thoughts of self-harm are reported, we assessed that with some additional questions, like: 'Is death something you've thought about recently?', 'Have things ever reached the point that you've thought of harming yourself?', 'When did you first notice such thoughts?', 'How often have those thoughts occurred (including frequency, severity, obsessional quality, controllability)?', 'How close have you come to acting on these thoughts?', 'How likely do you think it is that you will act on thoughts in the future?', 'Do you have guns or other weapons available to you?', 'How does the future look to you?', 'What things in your life would lead you to want to escape from life or be dead?'. If the patient's response reflects dissatisfaction or despair with life or a desire to escape it, this response can lead naturally into the more specific questions about whether the patient has had 


\section{Mental Health \& Human Resilience International Journal}

thoughts of death, or suicide. When such thoughts are elicited, it is important to focus on the nature, frequency, extent, and timing of them and to understand the interpersonal, situational, and symptomatic context in which they are occurring.

Sometime, the interview questions could be with no mentioning bad things which bother one's mind, or asking about plans for the future, such as: 'What things in your life make you to go on living?', 'If you have thoughts of selfharming, what would you do?', 'Would you do self-harm in presence of other people?', 'What you think will happen if you commit suicide?', 'How did you feel when your thoughts of self-harming are gone?', 'How your view of things changed since suicidal thoughts are gone?', 'Are there other people you would want to die with you?', 'Are there others who you think would be unable to go on without you?'. These questions target the patient's positive response, strength, and thoughts how to fight against his/her hopelessness and desperation. Regardless of the approach to an interview, not all individuals will report having suicidal ideation even when suicidal thoughts were present.

While assessing one's risk for suicide, it is advisable to obtain some collateral information and contact with significant others. The confidential nature doctor-patient relationship is a fundamental aspect of the therapeutic process. Consequently, the clinician will need to manage the tension between this requirement and the wish to act in the patient's best interest. Working with severely traumatised individuals, the clinician should establish good therapeutic alliance which could be a complicated process and involves trust, autonomy, initiative, and interplay [22].

However, in maintaining safe environment for the patient, significant other may need to be contacted to furnish information or carry out specific tasks such as suicidal risk (e.g., this, inter alia, will include removing firearms from home). If the clinician determines that the patient is (or is likely to become) dangerous to him- or herself or to others and the patient will not consent to interventions that aim to reduce those risks, then the clinician is justified in attenuating confidentiality to the extent needed to address the safety of the patient and others [23]. This decision requires clinical judgment in weighing the effects of breaching confidentiality on the therapeutic alliance against the potential safety risk for the patient and others.

\section{Results}

The main age of the participants in this study was 37.5 $(S D=11.7)$ years and more than half of them $(n=21$, $58.3 \%$ ) were men. The majority of individuals in the sample were married $(n=29,80.6 \%)$, unemployed $(n=$ $28,77.8 \%)$, and the vast majority $(n=34,94.4 \%)$ has been or still take prescribed medication. Only few of participants $(n=6,16.7 \%)$ reported alcohol consumption, but none reported illicit drugs use. None of the individuals reported any mental health problems before experiencing traumatic event(s), nor visiting psychiatrists or psychologist due to his/her mental health.

Results on the $B H S$ revealed no significant differences between participants who reported to referring doctor some level of suicidal behaviour and participants who did not mention it to their family doctor. Also, no significant differences were found between male and female participants who scored between 15-20 points on the $B H S$ (severe hopelessness).

However, there were significant differences on hopelessness scale between participants who suffered complex trauma syndrome due to the human-made traumatic events (rape, combat, violence, assault) and natural disaster (flooding, bushfire) as it is stated in Table 1. However, none of the 36 participants in this study reported attempted suicide since the trauma. The vast majority of the participants stated during the clinical interview ('Have you seriously thought about suicide?', 'Have you ever made plans to commit suicide?', 'Have you ever attempted suicide?') that their hopelessness was in particular high soon after the trauma having suicidal thoughts and plans, but they are thankful of supports from their families and friends that they did no attempt suicide.

\begin{tabular}{|c|c|c|}
\hline & Human-made trauma & Natural disaster \\
\hline$B H S$ (severe hopelessness) & $n=15, M=18.4$ & $n=21, M=15.2$ \\
\hline No suicidal ideation & $n=4,26.7 \%$ & $n=16,76.2 \%$ \\
\hline Reported suicidal thoughts & $n=8,53.3 \%$ & $n=4,19.0 \%$ \\
\hline Reported definite plan & $n=3,20.0 \%$ & $n=1,4.8 \%$ \\
\hline
\end{tabular}

Table 1: BHS scores and self-reported suicidality. 


\section{Mental Health \& Human Resilience International Journal}

Overall, 16 (44.4\%) participants reported suicidal behaviour after the trauma exposure. A considerable higher percentage of those who reported suicidal behaviour were victims of human-made trauma $(n=11$, $68.8 \%)$ vs $(n=5,31.2 \%)$, and also scored higher on BHS $(M=18.4, S D=3.4)$ vs $(M=15.2, S D=7.6)$. The low standard deviation of human-made trauma victims reveals consistency of their trauma symptoms. We assume that hopelessness results on the BHS by victims of natural disaster was mostly related to the losses (e.g., property, important memorabilia) than real dreadful feelings which could lead them to the suicidal behaviour.

The negative self-concept in trauma syndrome patients is also associated with the self-rejection. The patients not only see themselves as inferior, but they dislike themselves for it. They seem incapable of viewing themselves in any way other than in terms of deficiency, dejection, incompetency, pessimism, and hopelessness. If they recognise their trauma syndrome, they criticise themselves for being ill - they see their weakness as a chronical condition with no possibility to heal. As it is seen in Table 1, the higher level of hopelessness $(M=18.4$, $n=15$ ), and, subsequently, potentially higher risk for suicidal behaviour $(73.3 \%, n=11)$, is among patient who had experienced human-made disasters which potentially cause more severe and more complex trauma syndrome than natural disasters. Their detachment, including depersonalisation, derealisation and emotional numbing, had been devastating consequences for all aspects of the patients' life 'disruption and discontinuity in the normal integration of consciousness, memory, identity, emotion, perception, body representation, motor control, and behaviour' [20] p.291.

Similarly, it was significant differences between two groups of the participants in scores on CAPS, as it is presented in Table 2. It was evident that the CAPS total score is associated with severity of trauma syndrome symptoms, and the avoidance and numbing symptoms cluster is a direct link with social functioning impairment (including social detachment).

\begin{tabular}{|c|c|c|}
\hline & Human-made trauma & Natural disaster \\
\hline \multirow{2}{*}{$C A P S$} & $n=15, M=94.3$ & $n=21, M=63.2$ \\
& $S D=12.4$ & $S D=11.3$ \\
\hline
\end{tabular}

Table 2: Human-made trauma.

The results on CAPS are expected as clinicians (Courtois, Foa, Ford, Herman, Horowitz, Keane, van der Kolk, Wilson, Zepinic, Zlotnick, ...) are united in opinions that the human-made trauma causes more severe impact upon victims than the natural disasters. Due to the severity and complexity, the human-made traumatic event(s) usually causes complex trauma syndrome. Trauma affects all structures of the victim's self - one's image of the body; the internalised images of others; and one's values and ideals - and leads to a sense that coherence and one's goals are invaded, assaulted and systematically broken down [22].

The nature of any traumatised self by complex trauma is commonly 'an iceberg' for the clinician. The trauma may lead to a de-centering of self, loss of groundedness and sense of sameness, continuity and ego-fragility, leaving scars on patient's inner agency of the psyche [17]. Trauma syndrome patient's expectations usually have a negative cast and may occur in the form of a pictorial negativity, or as the catastrophic obsessive ruminations. Patient's anticipations of the future are generally an extension of what he views in his past, 'there-and-then' circumstances. As patient feels deprived, immobilised, or rejected, he visualises a future in which he is continually deprived, immobilised, or rejected - he is unable to view his current state as having any time limits or to consider the possibility of improvement.

Not only the patient's long-term forecast for future are in a negative territory, but his short-term predictions are similarly negative. When he awakes in the morning his thoughts are overwhelmed and he anticipates that every experience during new day will pose great difficulties and failures [24]. For the clinical importance, it is useful to make a distinction between negative predictions of the trauma syndrome patient and the fears of the anxious patient. When trauma syndrome patient looks ahead to the future, he could foresee a procession of future failures; this prospect did not produce any apprehension because he did not expect them to make him feel any worse than he feels now. The anticipated failures merely represent to patient the impossibility if ever feeling better and the futility of trying - he perceives himself as already damaged (defeated, deprived, or depreciated).

Traumatic injuries to the victim's sense of self can be quite catastrophic, often causing perception that one's unity and wholeness is lost forever - the complex trauma is analogous to a high-velocity bullet through the body tearing apart internal organs critical for survival. Complex trauma is a catastrophic experience of prolonged and often multiple traumas, and includes profound disturbances in interpersonal relationships and one's estrangement from others, pervasive mistrust, hostility and suspiciousness, feeling of emptiness, and altered sense of meaning and purpose of life $[6,15,17,25]$. The 


\section{Mental Health \& Human Resilience International Journal}

severely traumatised individuals are overwhelmed by the terror and hopelessness; their whole mechanisms for concerted, coordinated and purposeful activity are smashed. Severely traumatised individuals are sensitive to any impediments to their goal-directed activity and apt to interpret relatively trivial events as catastrophic or contributing to a substantial loss.

The clinical interview is an instrument to assess the patient's destructed self-structure by traumatic event(s). Having depleted or shattered self by the trauma, the patient's suicidal behaviour is 'Everything is worthless, killing myself is the only way out of a misery' - as a common patient's comment. Suicide can be emphasised as a conscious act of self-intended cessation; an act of selfinduced annihilation [26]. Due to the severe traumatic experience, the attachment is disturbed and, in posttrauma time, the victim has difficulties in bringing his psychic equilibrium into normal level, with no posttraumatic growth. Trauma syndrome makes patients prone to interpret neutral remarks about them as depreciation or directed against them in some way. Often patients with a complex trauma syndrome believe that other people have some derogative ideas about them (negative attributions).

Disrupted attachment ties and the compromise victim's self-integrity lead to the patient's unbearable emotional state: experience of hopelessness and emptiness. The damage to self-identity and integrity results in a victim's 'self-at-worst' state scenario: at this point the individual is at the most depleted level of the sense of self, with no safety net and thus no access to the emotional resources. Unable to impose 'survival skills' in post-trauma time, the severely traumatised individual attempts to escape the excruciating experience of this unbearable emotional state, and it is overwhelmed by the deadly and dangerous seeds of the development of his psychopathological condition - suicidal behaviours. Whatever else suicide is, it is a conscious act, but on the other hand, the driving force of an unconscious process. The patient's unconsciousness is an important construction in the understanding such complicated human act, as suicide is imposed by a mental constriction of existing emotional pain, hopelessness, and distortion [27].

The empty and shattered sense of self consists of one's passivity, depressiveness, and evident depletion in energy. Chronic traumatic condition, or complex trauma syndrome, causes an identity diffusion, fragility and feelings of discontinuity, with severely disrupted and/or shattered interpersonal relationships and existence. As a response to the trauma circumstances, many complex trauma survivors have a cluster of the specific symptoms: 'survivor syndrome' which shows its existence even long after the trauma is over. Trauma experience can lay dormant in the deeply unconscious state of the psyche for many years until a trigger event(s) awakes it. In the posttrauma time, the patient's relatedness and aliveness may still be based on 'survival skills', acquired during the traumatic experience. The patient's behaviour, thoughts, and emotions are still attached to the traumatic past, without an existing 'here-and-now' state [3].

This is in particular with combat-related PTSD in which ex-soldiers still experience 'there-and-then' state with reminders that increase risk of suicide or homicide. For example, in the USA rates of veteran deaths from suicide remain high - an average 20 veterans died from suicide each day, making the risk for suicide 21\% higher among veterans when compared to the civilian adults. Veterans constitutes $8.5 \%$ of the US population, however the veterans accounted for $18 \%$ of all death from suicide among US adults [28]. Further, some research [29] led us to the hypothesis that surviving an attempted suicide due to the other mental conditions (e.g., depression) may result in a trauma-like condition potentially triggering the development of PTSD as sequela of a traumatic suicide attempt.

The complex trauma syndrome patients not only interpret their traumatic experience as detracting from themselves in some way, but they concurrently devalue themselves. To sharpen the distinction between negative interpretation of the traumatic experience and negative view of the self, we can sometime see that even at the paranoid level - they may see others as thwarting or rejecting them, but unlike a trauma syndrome patient, the paranoid patient maintains positive concept of himself. The striking feature of the trauma syndrome patient is his tendency to generalise from a particular behaviour to the self-structure trait [17]. The supposed deficiency becomes so magnified that it occupies the individual's total selfconcept, and the self is seen nothing else than the 'self-atworst'. The patient seems incapable of viewing himself in any way other than in terms of functional deficiency for which the suicide is a solution to end a miserable life.

In the light of these findings, the risk of developing PTSD after an attempted suicide is aggravated by general PTSD risk factors such as severity and seriousness of the suicide attempt, presence of peritraumatic dissociation, a maladaptive coping style, as well as a history of other adverse life events [5,30]. Bill, et al. [31] found a high incidence of posttraumatic stress disorder in response to 


\section{Mental Health \& Human Resilience International Journal}

suicidal attempt in patients with a major depression. The incidence of PTSD was $46.7 \%$ among those who attempted suicide, which can be regarded as comparable to the high incidence rates usually observed after very severe traumatic event such as rape or physical abuse during childhood.

\section{Discussion}

The principal aims of this study were to evaluate relations between patient's trauma syndrome symptoms and suicidal behaviour. It is well-known that PTSD increases risk of depression and suicidal ideation in particular to those patients who are victims of humanmade traumatic event(s). The estimated suicide among severely traumatised individuals is controversial considering that other factors (alcoholism, drugs addiction, unemployment, etc.), are also contributing to patients' suicidal behaviour. However, many studies revealed that, for example, there were more deaths of Vietnam veterans by suicide after the war than deaths in combat. Stevens, et al. [32] stated that suicide risk could be higher in those with trauma syndrome due to the symptoms of PTSD (e.g., intrusive memories, irritability, anger, and poor impulse control) and/or commonly comorbid psychiatric condition (e.g., major depression, drug, and alcohol abuse). Clinicians [3,6,11,15,25] are agreed that PTSD caused by assaultive violence more potentiates higher risk for suicide than the PTSD caused by the natural disasters.

The findings in this study are consistent with our hypothesis that clinical symptoms of trauma syndrome and variables which reflect the 'self-at-worst' and functional impairment, are closely associated with patient's suicidal behaviour. Based on previous findings $[3,5,6,7,11,16,22,23]$, it was hypothesised that symptoms of complex PTSD would be a strong predictor of the patient's suicidal behaviour. We found that depleted sense of self, hopelessness, and social detachment were directly associated with suicidal behaviour. Thus, it appears that, in complex trauma syndrome, the attention to suicidal behaviour should be an ultimate part of assessment and throughout the entire treatment.

Clinical models of suicidality $[8,19,20,23,27]$ propose that the negative dysfunctional appraisals, such as feelings of defeat, hopelessness, and entrapment, are the overriding factors that drive suicidal behaviour. Consistent with research findings [3,4,11,15,26,31], the complex PTSD symptoms contribute to suicidal behaviour through increasing the severity of symptoms of the depression (social withdrawal, sleep problems, loss of energy, feelings of worthlessness, diminished ability to concentrate, recurrent thoughts of death). Additionally, the avoidance and numbing symptoms cluster contributed also to suicidal behaviour in complex PTSD patients because it is resulted in an impaired social functioning due to the avoidance and numbing symptoms associated with severe re-experiencing symptoms of trauma.

In other words, the higher the severity of the reexperiencing symptoms cluster (e.g., memories, flashbacks, nightmares) the more likely it is for individuals to adopt cognitive and behavioural avoidance strategies together with other dysfunctional strategies to cope with the distressing intrusions that clearly have an adverse effect on their functioning [2]. Perceived threat to life during the traumatic event(s) had a direct impact on the severity of PTSD symptoms and thus contributed indirectly (sometime even directly) to the suicidal behaviour. Surprisingly, neither the DSM or the ICD has examined the role of such negative dysfunctional appraisals in the development of suicidal behaviour (for diagnostic criterion) in complex trauma syndrome $[14,18,22]$. A primary focus of the research on the complex trauma syndrome (complex PTSD) and suicidal behaviour in the future should be to rectify this limitation.

Wilcox, et al. [33], as well as other clinicians, grouped traumatic events into two broad categories: the events that involve assaultive violence and those that do not. The first category (assaultive violence) includes personally experiencing physical assault (e.g., being attacked, slapped, kicked, beaten), assault with a weapon (e.g., being shot, stabbed, threatened), sexual assault (e.g., rape, attempted rape, battery, made to perform any type of sexual act), other unwanted or uncomfortable sexual experience, combat or exposure to a war zone in the military or as a civilian, captivity (e.g., being kidnaped, abducted, held hostage, prisoner of war).

Other category of the traumatic events includes nonassaultive trauma category such as natural disaster (e.g., flood, bushfire, hurricane, tornado, earthquake), fire or explosion, transportation accident (e.g., car, boat or train accident), serious accident at work, home, or during recreational activity, exposure to toxic substances (e.g., dangerous chemicals, radiation), life threatening illness or injury, witnessing severe human suffering (e.g., homicide, suicide), serious injury, harm, or death caused by someone else, witnessing any of the assaultive violence events (not personally experiencing them). They $[2,3,9,12,15,33]$ found that a history of PTSD involving exposure to assaultive violence is a strong predictor of 


\section{Mental Health \& Human Resilience International Journal}

one subsequent suicide attempt even after controlling for the presence of psychiatric comorbidity before first exposure to trauma.

Jakupcak, et al. [34] found that the war veterans who screen positive for PTSD are four times as likely to report the suicidal ideations as their peers without PTSD. Pompili, et al. [35] conducted a systematic review of 80 empirical studies and concluded that having a history of the PTSD diagnosis is linked to significant morbidity and mortality, including increased risk for suicidal ideation, attempts, and completed suicide in war veterans. Loncar, et al. [36] found that symptoms of PTSD and higher risk for suicide among war veterans may persist for and occur many years after their deployment. Jaksic, et al. [37] found that the war veterans with combat-related PTSD and comorbid depressive disorder seem to be more suicidal and characterised by different personality profile than those suffering from PTSD alone.

Zepinic [3] stated that combat-related PTSD leads to loss of identity, guilt, a sense of failure, and shame. The most prominent symptoms of the combat stress reactions were recurrence of traumatic scenes and thoughts, sleep difficulties, loss of interest in previous enjoyable activities, cognitive dysfunctions, hopelessness, worthlessness, and suicidal ideation. Suicidal patients reported their wish to terminate personal discomfort and found suicide as a viable method of achieving that. Those severely traumatised reported also helplessness, feelings of detachment, loss of the wish to live, and a sense of foreshortened future. For them, the post-war time has been persistent re-experience of recurrent and intrusive distressing recollections of the war-time that they have been exposed to or witnessed, including an actual or threatening death or serious injury, or a threat of the physical integrity of self or others. It is common that warrelated PTSD patients comment that everything is worthless, although they very rare will report suicidal thoughts or attempts during combat or when being prisoners of war.

Suicidality, which sometimes served as a form of resistance during imprisonment, may persist long after release, when it no longer serves any adaptive purpose [15]. Those who had been subjected to torture often bear the psychological scars of their captivity. Long after trauma is over their classic trauma syndrome causes profound alterations in their relations with other people or even with their own self. For example, Bruno Bettelheim, Vienna-born psychologist and psychoanalyst himself survivor of Nazi concentration camp during the WW II, did not attempt suicide while in the camp; but he did later on in 1990 at the age of 86 , as did the author Primo Levi in 1987, a survivor of Auschwitz.

In the words of the Holocaust survivor Levi [38]: 'We have learnt that our personality is fragile, that it is in much more danger than our life; and we the old wise ones, instead of warning us 'remember that you must die', would have done much better to remind us of this greater danger that threaten us'. Putnam [39] stated that the more severe trauma is, the greater the likelihood that an individual will be driven into an altered state of all dimensions of consciousness: time, thought, body, and emotion. In case of severe trauma (complex trauma syndrome), the mind/body connection is often severed, leading to the subjective experience of feeling partially or fully detached from one's body, or alternatively, as if one's body does not belong to oneself [40].

In his research, Zepinic [3] using adapted MMPI (form 201) to assess 20 war-prisoners from former Yugoslavia five years after being released, found that patients showed typical $D$-Pt profile (depression-psychasthenia). The $D$-Pt profile which was found among the warprisoners is characterised by fears, sadness, decreased emotional and working energy, low level of spontaneity and impulse control, disposition to the self-accusation and self-blame, worthlessness, helplessness, high level of anxiety with psychosomatic and/or physical reactions, difficulty concentrating, insomnia, libido problems, loss of appetite, dichotomy in diagnosis (neurotic-psychotic, endogenic-reactive), mental and/or physical fatigue, and restlessness.

In our study, while using instruments stated above (BHS, CAPS, SCID, clinical interview), we found that the hopelessness was a key variable in any suicidal behaviour which can be conceptualised as a system of cognitive schemata having a common element of negative expectations of the future. Patients reported their future expectations as a darkness, not getting good things (things not working out), the future being uncertain, and a general lack of enthusiasm, happiness, good times or anything related to hope. Loss of motivation and mental energy included absence of all positive thinking, deciding not to want anything, and not to trying to get something what has been enjoyable and welcome before the traumatic event(s). Severely traumatised individuals believe they would not get well, nor solve personal problems ('I lost myself', or 'I am not myself anymore'), would have nothing to look forward to, or would fail to achieve personal goals, and view the future with a pessimism. 


\section{Mental Health \& Human Resilience International Journal}

Using SCID with participants in this study, we recognise characteristic varieties of the stress syndrome which are highly potential risk for the patients' suicidal behaviour: $80 \%$ patients reported memory/concentration problems, 64\% reported severe sleep disturbances, $64 \%$ reported irritability, $60 \%$ reported restricted expectation about the future, $55 \%$ lost interest in previously enjoyable activities, $50 \%$ reported having nightmares, and $45 \%$ detachment from others (friends, or even family members). Further, they reported clinically significant disruption of mental life with problems that appeared after trauma experience: hostile and mistrustful attitude toward the world, social withdrawal and estrangement, feelings of emptiness, and chronic feeling of being 'on the edge', as if constantly threatened. It is critical for the clinicians to understand the patient's subjective experience of suicidal behaviour and its relations to the complex trauma syndrome.

The clinicians who threat complex trauma syndrome patients should be well aware of the suicidal risk factors from two perspectives. Firstly, if a patient is speaking of his/her suicidal thoughts or any suicidal risk behaviour, or is in other ways thought to be endangering himherself, it must be considered whether or not the patient might yet be undiagnosed for psychiatric disorder. Secondly, for a patient with the disorders of these types, the clinician should be extremely vigilant if the patient shows any sign of self-destructive behaviour. Patients who may have such diagnosis must be referred for psychiatric assessment with, if necessary, short hospitalisation and a proper inpatient management. The clinician should also assess the functioning of family members and their ability to care for and control the patient during times of crisis.

\section{References}

1. Harned MS, Jackson CS, Comptois KA, Linehan MM (2 010) Dialectic Behaviour Therapy as a Precursors to PTSD Treatment for Suicidal and/or Self-Injuring Women with Borderline Personality Disorder. Journal of Traumatic Stress 23(4): 421-429.

2. Panagioti M, Gooding PA, Tarrier N (2009) Posttraumatic stress disorder and suicidal behaviour: A narrative review. Clinical Psychology Review 29(6): 471-482.

3. Zepinic V (2001) Suicidal Risk with war-related posttraumatic stress disorder, chapter 14, In: Raphael B, Malak AE (Eds.), Diversity and Mental Health in Challenging Times. TCMHC, Sydney.

Vito Z. Suicidal Behaviour in Complex Trauma Syndrome. Ment Health Hum Resilience Int J 2020, 4(1): 000136.
4. Zepinic V (2015) Persistence of 'Survival Skills' as a Risk Factor for Suicide in Severely Traumatised Individuals. International Journal of Emergency Mental Health and Human Resilience.

5. Zepinic V (2009) The sense of self and suicide. Dynamische Psychiatrie, pp: 5-6.

6. Zepinic V (2016) Disintegration of the Self-Structure Caused by Severe Trauma. Psychology and Behavioural Science 5(4): 83-92.

7. Baumeister RF (1990) Suicide as Escape from Self. Psychological Review 97(1): 90-113.

8. Yufit RL, Lester D (2005) Assessment, Treatment and Prevention of Suicidal Behaviour.

9. Van der Hart 0, Dorahy MJ (2009) History of the concept of dissociation. In: Dell PF, O Neil JA, (Eds.): Dissociation and the Dissociative Disorders. Routledge, New York.

10. Zepinic V (2017) Trauma-focused Dynamic Therapy model in treating complex psychological trauma. Psychology 8: 2059-2101.

11. Ulman BR, Brothers D (1988) The Shattered Self. A psychoanalytic study of trauma.

12. Van der Kolk BA, MacFairlane A, Weiseath L (1996) Traumatic Stress. The Guilford Press.

13. Wolf SE (1998) Treating the Self. The Guilford Press.

14. Goodman M (2012) Complex PTSD is on the Trauma Spectrum: Comment on Resick et al. (2012). Journal of Traumatic Stress 25(3):254-255.

15. Herman J (1997) Trauma and Recovery. Basic Books Ney York.

16. Courtois AC (2004) Complex trauma, complex reactions: Assessment and treatment. Psychotherapy: Theory, Research, Practice, Training 41(4): 412-425.

17. Zepinic V (2018) The Self and Complex Trauma. Austin Macauley Publishers, London.

18. Herman J (2012) CPTSD is a Distinct Entity: Comment on Resick et al. (2012). Journal of Traumatic Stress 25(3): 256-257.

19. Beck AT, Steer RA (1993) Beck Hopelessness Scale Manual. Harcourt Brace San Antonio. 


\section{Mental Health \& Human Resilience International Journal}

20. Vihang N, Vahia (2013) Diagnostic and Statistical Manual of Mental Health Disorders (DSM-5). Washington DC 55(3): 220-223.

21. Blake D, Weathers F, Nagy F, Kaloupek D, Gusman F, et al. (1995) The development of a Clinician Administrated PTSD Scale. Journal of Traumatic Stress 8(1): 75-90.

22. Zepinic V (2011) Hidden Scars: Understanding and Treating Complex Trauma. Xlibris Publishing, London.

23. American Psychiatric Association (2003) Practice Guideline for the Assessment and Treatment of Patients with Suicidal Behaviour, Washington DC Am J Psychiatry 160(11): 1-60.

24. Zepinic V (1997) Psychosocial characteristics of warrelated Posttraumatic Stress Disorder. chapter 10, In: Ferguson B, Barnes D (Eds.), Perspectives on Transcultural Mental Health. TCMH, Sydney.

25. Courtois CA, Ford JD (2009) Treating Complex Traumatic Stress Disorder. The Guilford Press, New York.

26. Zepinic V (2018) Survival Skills as a risk factor for suicide in complex PTSD. Abnormal Psychology and Clinical Psychiatry 1(1): 004.

27. Shneiden ES (1985) Definition of suicide, Wiley \& Sons, New York.

28. Lutwack N, Dill C (2014) Letter to the Editor. Military Medicine, pp: 182.

29. Yehuda R (2009) Status of glucocorticoid alterations in post-traumatic stress disorder. Annals of New York Academy of Science 1179: 56-69.

30. Brewin CR, Andrews B, Valentine JD (2000) Metaanalysis of risk factors for posttraumatic stress disorder in trauma-exposed adults. Journal of Consulting and Clinical Psychology 68(5): 748-766.

31. Bill B, Ipsch L, Lucae S, Pfister H, Maragkos M, et al. (2 012) Attempted Suicide Related Posttraumatic Stress Disorder in Depression-An Explanatory Study. Suicidology 3: 138-144.
32. Stevens D, Wilcox HC, MacKinnon DF, Mondimore FM, Schweizer B, et al. (2013) Posttraumatic Stress Disorders Increases Risk for Suicide Attempt in Adults with Recurrent Major Depression. Depression and Anxiety 30(10): 940-946.

33. Wilcox HC, Storr CL, Breslau N (2009) Posttraumatic stress disorder and suicide attempts in a community sample of urban American young adults. Archive of General Psychiatry 66(3): 305-311.

34. Jakupcak M, Cook J, Imel Z, Fontana A, Rosenheck R, et al. (2009) Posttraumatic stress disorder as a risk factor for suicide ideation in Iraq and Afghanistan war veterans. Journal of Traumatic Stress 22(4): 303306.

35. Pompili M, Sher L, Serafini G, Forte A, Innamorati M, et al. (2013) Posttraumatic stress disorder and suicide risk among veterans: A literature review. Journal of Nervous Mental Disorders 201(9): 802812.

36. Loncar M, Plasc ID, Bunjevac T, Hrabac P, Jakšić N, et al. (2014) Predicting symptom clusters of posttraumatic stress disorder (PTSD) in Croatian war veterans: The role of socio-demographics, war experiences and subjective quality of life. Psychiatria Danubina 26(3): 231-238.

37. Jaksic N, Margetic AB, Marcinko D (2017) Comorbid depression and suicide ideation in patients with combat-related PTSD: The role of temperament, character, and trait impulsivity. Psychiatria Danubina 29(1): 51-59.

38. Levi P (1961) Survival in Auschwitz: The Nazi Assault on Humanity. Collier, New York.

39. Putnam FW (1996) Dissociation in children and adolescents: A developmental perspective. Guilford Press, New York.

40. Lanius RA (2015) Trauma-related dissociation and altered states of consciousness: a call for clinical, treatment, and neuroscience research. European Journal of Psychotraumatology 6: 27905. 